Imaging of soft matter with tapping-mode atomic force microscopy and non-contact-mode atomic force microscopy

This article has been downloaded from IOPscience. Please scroll down to see the full text article. 2007 Nanotechnology 18084009

(http://iopscience.iop.org/0957-4484/18/8/084009)

The Table of Contents and more related content is available

Download details:

IP Address: 140.112.113.225

The article was downloaded on 26/06/2009 at 11:07

Please note that terms and conditions apply. 


\title{
Imaging of soft matter with tapping-mode atomic force microscopy and non-contact-mode atomic force microscopy
}

\author{
Chih-Wen Yang ${ }^{1,2}$, Ing-Shouh Hwang ${ }^{1,3}$, Yen Fu Chen ${ }^{1}$, \\ Chia Seng Chang ${ }^{1}$ and Din Ping Tsai ${ }^{2}$ \\ ${ }^{1}$ Institute of Physics, Academia Sinica, Nankang, Taipei, Taiwan, Republic of China \\ ${ }^{2}$ Department of Physics, National Taiwan University, Taipei, Taiwan, Republic of China \\ E-mail: ishwang@phys.sinica.edu.tw
}

Received 13 July 2006, in final form 1 September 2006

Published 18 January 2007

Online at stacks.iop.org/Nano/18/084009

\begin{abstract}
In this work, we take topographic images of DNA molecules and nanometre-sized polystyrene spheres in air as well as in water using an atomic force microscope (AFM). We would like to compare the applied forces and the resolution for images taken with the amplitude-modulation (AM) detection, or the tapping mode, and the frequency-modulation (FM) detection, or the non-contact (NC) mode. Experiments for these two modes are carried out on the same area of the samples, with the same tip, under the same environment, and with the same oscillation amplitude. Our experiments indicate that, in the FM mode, the tip exerts a very gentle force on soft materials and provides a height measurement close to the true value. In the AM mode, the tip exerts a stronger force on soft materials and causes their deformation, especially in the liquid environment. The resolution of the FM mode is about the same as that of the AM mode for operation in air, but the former is significantly superior to the latter in water. We thus conclude that the FM mode can obtain images with a higher resolution and provides a sufficient sensitivity to image the true and fine structure of soft matter on surfaces.
\end{abstract}

\section{Introduction}

Atomic force microscopy has been widely used to investigate structures and mechanical properties of materials on surfaces. Very high spatial resolution can be achieved regularly on hard surfaces. In particular, atomic resolution on flat surfaces of hard materials has been demonstrated in vacuum by many research groups $[1,2]$. Nevertheless, there is an increasing interest in applying atomic force microscopy for imaging soft materials, especially biological samples in liquid. The goal is to achieve high-resolution images of biological samples in physiological conditions, which could unravel many puzzles

3 Author to whom any correspondence should be addressed. in life science. Atomic force microscopy experiments in an aqueous solution are of major importance since only this environment allows the study of native biological processes. However, biological molecules are usually delicate and soft. For operation in the contact mode, the AFM tip tends to damage or dislodge them during scanning. If the AFM is operated at a weaker tip-sample force, the resolution degrades and the AFM may not be under a stable operation. It is, therefore, a great challenge to image biological samples, especially in liquid.

The tapping mode [3] has been one of the most widely used methods in atomic force microscopy. It is considered to be more gentle than the contact mode in imaging soft materials or nanomaterials that are weakly attached to the substrate. In 
the tapping mode, the cantilever (driven by a piezoelectric actuator) vibrates at or near its resonance frequency. Upon approaching the sample, the tip briefly touches, or taps, the surface at the bottom of each swing, resulting in a decrease in oscillation amplitude. The feedback keeps this amplitude at a preset value and a topographic image of the sample surface can be obtained. This mode is also named as the amplitudemodulation (AM) mode. Its force sensitivity is dependent on the quality factor ( $Q$-factor) of the oscillating cantilever.

An alternative method is the frequency-modulation (FM) detection scheme $[4,5]$, also known as the non-contact mode. FM-atomic force microscopy can resolve individual atoms on several hard, flat surfaces in vacuum. In this mode, the cantilever is vibrated at the resonant frequency. When the tip is close enough to interact with the sample surface, the force gradient acting on the tip can lead to a shift in the resonance frequency of the oscillating cantilever. It has been derived that the frequency shift, $\Delta f$, is proportional to $\left(-\partial F_{\mathrm{ts}} / \partial z\right)$, where $F_{\mathrm{ts}}$ is the interaction force between the tip and the sample [1,5]. The feedback keeps the frequency shift at a preset value and a topographic image of the sample surface can be obtained. This mode has been shown to achieve a very high sensitivity of the force gradient in vacuum because of the much enhanced $Q$ value of the oscillating cantilever [1, 2, 5-9]. For years, it was not expected that this mode could be operated in water because the $Q$-value decreases significantly due to the hydrodynamic interaction between the cantilever and the liquid. Recently, atomic resolution in liquid was reported with the FM mode by Fukuma et al [10-12].

In this work, we would like to make an objective comparison between the AM and the FM modes in imaging soft materials in air as well as in water. We find that the FM mode performs better than the AM mode in terms of the applied force and the resolution. The difference is even more prominent in water. If the operation conditions of the AM mode are carefully chosen, the tip does not cause damage on DNA molecules, but it still causes deformation of the sample, especially in water. With the FM mode, we can image soft samples with a high resolution. Most importantly, the tip-sample interactions are so gentle that little deformation is detected.

\section{Experimental details}

Experiments are performed with a commercial beam-deflection AFM (DI NanoScope III(a), Digital Instrument, Santa Barbara, $\mathrm{CA})$. The AM and the FM modes are implemented with the combination of an easyPLL unit (Nanosurf $^{\circledR}$ easyPLL plus system) through a Signal Access Module (Digital Instrument, Santa Barbara, CA). A schematic diagram for our setup is shown in figure 1. For imaging in water, a drop of deionized water is introduced between the sample and an anti-reflection glass plate. We use silicon cantilevers with integrated tips (MikroMasch AFM cantilevers). The spring constant $k$ is $\sim 1 \mathrm{~N} \mathrm{~m}^{-1}$. The scan rate in all the image acquisitions is $0.5 \mathrm{~Hz}$. With this rate, we can easily achieve stable operation of the AFM and obtain good topographic images.

Lambda DNA (duplex DNA, BioLabs ${ }^{\circledR}$ Inc., 48502 base pairs) and polystyrene spheres are investigated in this study. The duplex DNA was isolated from bacteriophage lambda (cl857ind 1 Sam 7). For imaging in air, duplex DNA is
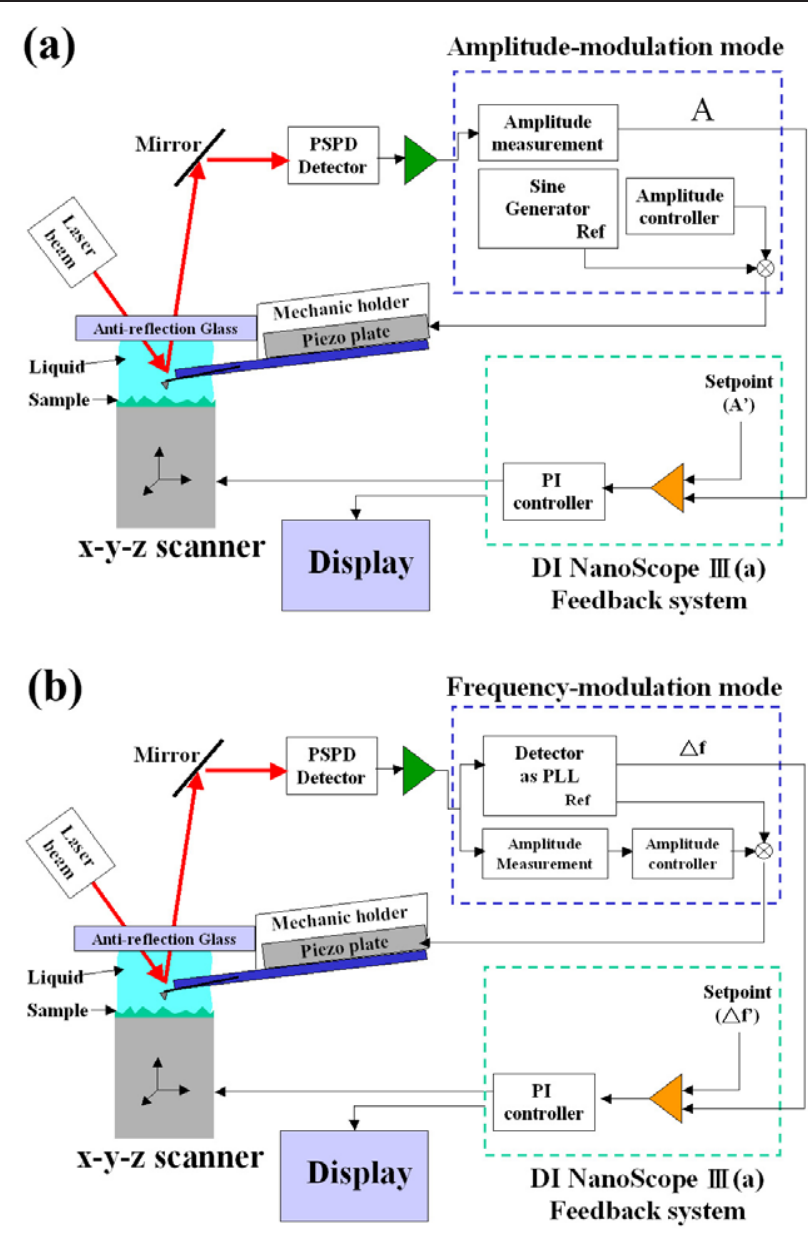

Figure 1. Schematic diagrams for amplitude detection (a) and frequency detection (b)

physically adsorbed onto a highly ordered pyrolytic graphite (HOPG) surface by placing a $20 \mu 1$ drop of DNA solution $\left(0.5 \mu \mathrm{g} \mathrm{ml}^{-1}\right)$ on the HOPG surface for $5 \mathrm{~min}$. The solution is removed with a filter paper. Then the sample is placed in a dry box for about an hour before AFM imaging. In water, we choose a mica surface for imaging duplex DNA because DNA does not adsorb on HOPG surfaces. However, the mica surface and the DNA are negatively charged in water. We use $\mathrm{Ni}^{2+}$ ions to immobilize DNA on the surface. $20 \mu \mathrm{l}$ of a mixed

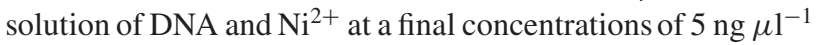
and $3 \mathrm{mM}$, respectively, is dropped onto a freshly cleaved mica surface. After waiting for $5 \mathrm{~min}$, this sample is placed onto the AFM stage and $5 \mathrm{ml}$ of pure water is introduced between the sample and the anti-reflection glass plate. AFM images are acquired after waiting for $\sim 15 \mathrm{~min}$.

Another sample is polystyrene spheres (Duke Scientific Corp.) on the HOPG substrate. We first disperse polystyrene spheres of 20 and $50 \mathrm{~nm}$ in diameter in ultra-pure water. The HOPG substrate is then dipped into this solution for $15 \mathrm{~min}$. The sample is rinsed with deionized water and left to dry before being placed on the AFM stage for imaging.

In this study, we start the imaging with the AM mode and adjust the working amplitude of the cantilever to get a stable operation and the best image quality of the DNA molecules or polystyrene spheres. The working frequency of 

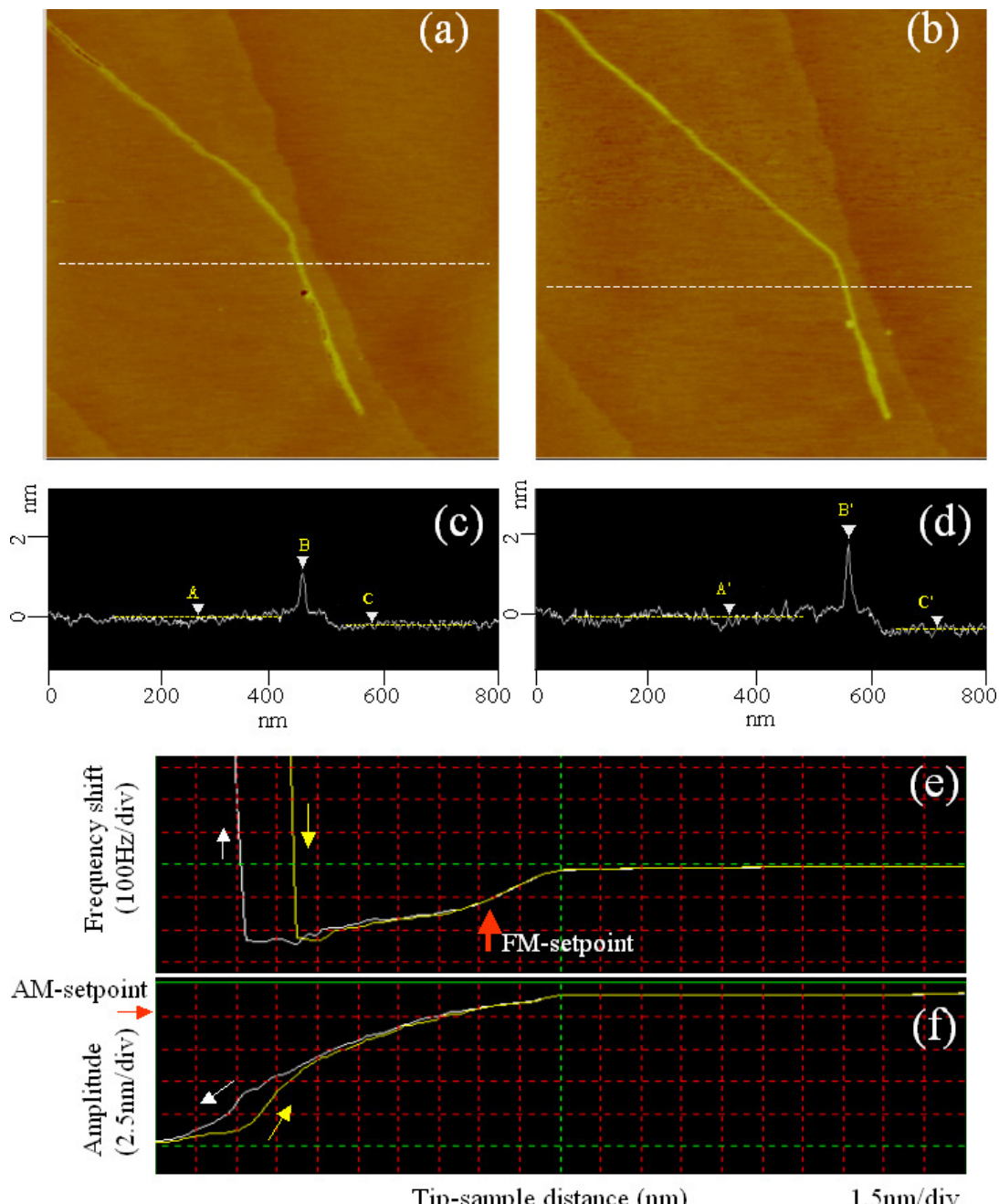

Figure 2. Measurements of duplex DNA on an HOPG surface in air. Scan area: $800 \mathrm{~nm} \times 800 \mathrm{~nm}$. The resonance frequency of the cantilever is $\sim 83.95 \mathrm{kHz}$ and the $Q$-factor is $\sim 142$. (a) Topographic image taken with the AM mode $\left(\mathrm{A}_{\text {free }}=13.6 \mathrm{~nm}, \mathrm{~A}^{\prime}=11.2 \mathrm{~nm}\right)$. (b) Topographic image taken with the FM mode $(\Delta f=-102 \mathrm{~Hz}, \mathrm{Amp}=11.2 \mathrm{~nm}$ ). (c) Height profile of duplex DNA along the white line in (a). (d) Height profile of duplex DNA along the white line in (b). (e) Approach/retract curve of the frequency shift of the cantilever versus the tip-sample distance. The setpoint of the frequency shift is indicated by a red arrow. (f) Approach/retract curve of the oscillation amplitude of the cantilever versus the tip-sample distance measured simultaneously with (e).

the cantilever is set at its intrinsic resonance frequency, which can be measured when the tip is far away from the sample. From our experience of operation with the AM mode, the above working parameters are at or near the optimum condition to achieve the best image quality. When we switch to the FM mode, the constant-amplitude mode is employed to maintain the oscillation amplitude of the cantilever equal to the working amplitude operated in the AM mode. For the measurement of the frequency shift versus the tip-sample spacing, the constantexcitation scheme is used, so that the oscillation amplitude versus the tip-sample spacing can be simultaneously obtained. In the constant-excitation scheme, an oscillation signal with a constant amplitude is applied to the piezo-element that drives the motion of the cantilever.

\section{Results and discussion}

Figures 2(a) and (b) show two topographic images of a duplex DNA on an HOPG surface, taken in air. The images in figures 2(a) and (b) are acquired with the AM mode and the FM mode, respectively. Since DNA is very fragile and has a weak adhesion to the substrate, the working amplitude of the cantilever in the AM operation should be carefully chosen to achieve stable operation, good resolution, and minimal loading force. We choose the free oscillation amplitude at $13.6 \mathrm{~nm}$, and the working amplitude is set at $11.2 \mathrm{~nm}$. For the FM mode, the oscillation amplitude is also set at $11.2 \mathrm{~nm}$.

Figure 2(c) shows a height profile along the white dashed line in figure 2(a). The average height of the upper terrace (lower terrace) is shown with a yellow dashed line in figure 2(c). Point A (C) indicates a position on the line profile that has a height equal to the average height of the upper (lower) terrace. The height difference between points $\mathrm{A}$ and $\mathrm{C}$ is $\sim 0.33 \mathrm{~nm}$, which is the single atomic step height on the HOPG surface. This step height can serve as a standard for calibration of the height measurement. The height of the duplex DNA molecule can be estimated from the height difference between points $\mathrm{A}$ and $\mathrm{B}$, which is $\sim 1.28 \mathrm{~nm}$. 
Figure 2(d) shows the corresponding height profile along the dashed line in figure 2(b). The same procedure as in figure 2(c) has been applied here. The measured HOPG step height between points $\mathrm{A}^{\prime}$ and $\mathrm{C}^{\prime}$ is $\sim 0.39 \mathrm{~nm}$, and the measured height of the duplex DNA molecule (between points $\mathrm{A}^{\prime}$ and $\mathrm{B}^{\prime}$ ) is $\sim 1.96 \mathrm{~nm}$. This indicates that the height of the duplex DNA molecule measured with the FM mode is significantly higher than that with the AM mode.

It is well known that height measurements based on the response of piezoelectric materials may have an uncertainty as high as $10-15 \%$, mainly due to the nonlinear characteristics of the response [13]. Therefore, it is important to calibrate the measurements with a thickness standard, such as the atomic layer thickness of a known material. The theoretical value for the layer thickness of the HOPG is $0.35 \mathrm{~nm}$. If we assume that our HOPG sample has an atomic step height of $0.35 \mathrm{~nm}$ and that the topography imaging does not cause any distortion in the height measurement of the HOPG atomic step, the calibrated DNA height is $1.36 \mathrm{~nm}$ for the AM mode and $1.76 \mathrm{~nm}$ for the FM mode.

The diameter of duplex DNA has been measured with $\mathrm{x}$-rays to be $\sim 2.0 \mathrm{~nm}[14]$. This value is very close to that measured with the FM mode here. The smaller height value obtained with the AM mode indicates that the soft DNA molecule is under a larger loading force. In contrast, with the FM mode, the tip exerts a smaller force on the molecule and the measured height is closer to the true value.

It is widely known that the DNA height measured with the AM mode in air is always less than the expected value of $2 \mathrm{~nm}$. Numerous measurements reported in the literature were even much smaller than $1 \mathrm{~nm}$ [15-18]. The discrepancy was attributed to several factors, including sample deformation by the loading force of the tip, salt deposition, strong electrostatic attraction between the molecule and the substrate, and dehydration of the molecule. In comparison, the measured height of 1.28 or $1.36 \mathrm{~nm}$ (calibrated value) in our AM-AFM is much closer to $2 \mathrm{~nm}$ than those reported in the literature. In addition, the salt deposition and strong attractive interaction of the molecule with the substrate do not occur in our case, because no salt is added and an HOPG substrate, instead of mica, is used. Therefore, the reduced height of our AM mode can be attributed to the loading force of the tip. Furthermore, calibration with an atomic step height as a standard, as shown in this work, is lacking in the literature.

Figure 2(e) shows the measured approach/retract curve of the frequency shift versus the tip-sample spacing for the FM mode. Meanwhile, the oscillation amplitude versus the distance is simultaneously recorded, as shown in figure 2(f). Figure 2(e) shows that, during approach, the resonance frequency of the cantilever exhibits a gradual decrease first and reaches a minimum, then a sharp increase occurs below a certain spacing. The decrease in the resonance frequency (negative frequency shift) indicates net-attractive interactions between the tip and the surface during initial approach. At the spacing where a sharp increase in the resonance frequency occurs, the tip-surface interactions suddenly turn repulsive. This force curve shows a hysteresis in the repulsive regime, but no hysteresis is seen in the attractive regime. The topographic image shown in figure 2(b) is taken at the frequency shift, the setpoint, indicated by a red arrow in figure 2(e). Therefore, it is taken in the non-contact (attractive) regime.
Figure 3 shows results of a similar experiment, except that it is carried out in water. The sample is duplex DNA on a mica surface. Figures 3(a) and (b) show two topographic images, which are acquired with the AM mode and the FM mode, respectively. We note that the FM mode is operated in the repulsive regime, which will be explained later. Figures 3(c) and (d) show height profiles along the dashed line in figures 3(a) and (b), respectively. For the AM mode operation, we choose the free oscillation amplitude at $6.2 \mathrm{~nm}$, and the working amplitude is set at $4.8 \mathrm{~nm}$, which is also the amplitude used in the FM mode. This working amplitude is smaller than that in figure 2 , because there is no capillary force in the liquid environment.

If we compare the images of duplex DNA in figures 3(a) and (b), it is evident that the FM mode provides a clearer image with more fine features than the AM mode. Most surprisingly, the measured height profiles across the DNA molecule exhibit a large difference between the two modes. The height measured with the FM mode is $\sim 2.42 \mathrm{~nm}$ (between points $\mathrm{A}^{\prime}$ and $\mathrm{B}^{\prime}$ ), but only $\sim 1.26 \mathrm{~nm}$ (between points $\mathrm{A}$ and B) is obtained with the AM mode. Clearly, the FM mode is much better than the AM mode in terms of the resolution and the loading force on the sample.

Because we cannot find atomic steps on this mica surface, calibration of the height measurements is not carried out in this case. However, the height of $2.42 \mathrm{~nm}$ obtained with the FM mode is significantly larger than $2 \mathrm{~nm}$, even with consideration of an uncertainty of $10-15 \%$. We do not know exactly which factors cause this enlarged value. Perhaps the hydration of the molecule in water could lead to this enlarged value. Also, the local charge distribution on the molecule may be different from that of the substrate and thus the electrostatic forces for tipDNA and tip-substrate are different. Further investigation is needed in the future.

Figure 3(e) shows the measured approach/retract curve of the frequency shift versus the tip-sample spacing for the FM mode. Meanwhile, the oscillation amplitude versus the distance is simultaneously recorded, as shown in figure 3(f). In contrast with the case in air, there is no attractive regime in water. The resonance frequency exhibits a gradual increase for the initial approach and a sharp increase below a certain tip-sample separation. The approach/retract curve shows little hysteresis. Figure 3(f) indicates that, during approach, the oscillation amplitude does not change until the point where a sharp increase in the resonance frequency occurs.

The image seen in figure 3(b) is taken at a constant frequency shift of $+94 \mathrm{~Hz}$, which is indicated by a red arrow in figure 3(e). Even though the FM mode is operated in the repulsive regime, the loading force of the AFM tip on the sample appears to be very small, so the height of duplex DNA is close to its true value. In comparison, with the AM mode, the tip does exert a strong force on the sample, such that the DNA molecule is deformed significantly. Therefore, its measured height is reduced by half and the width is enlarged.

Another sample we use is polystyrene spheres deposited on an HOPG surface. Two different sizes of polystyrene sphere $(20$ and $50 \mathrm{~nm}$ ) are deposited, because they allow us to determine the resolution of the topographic images. Figures 4(a) and (b) show two topographic images taken in air with the AM and the FM modes, respectively. Both sizes 

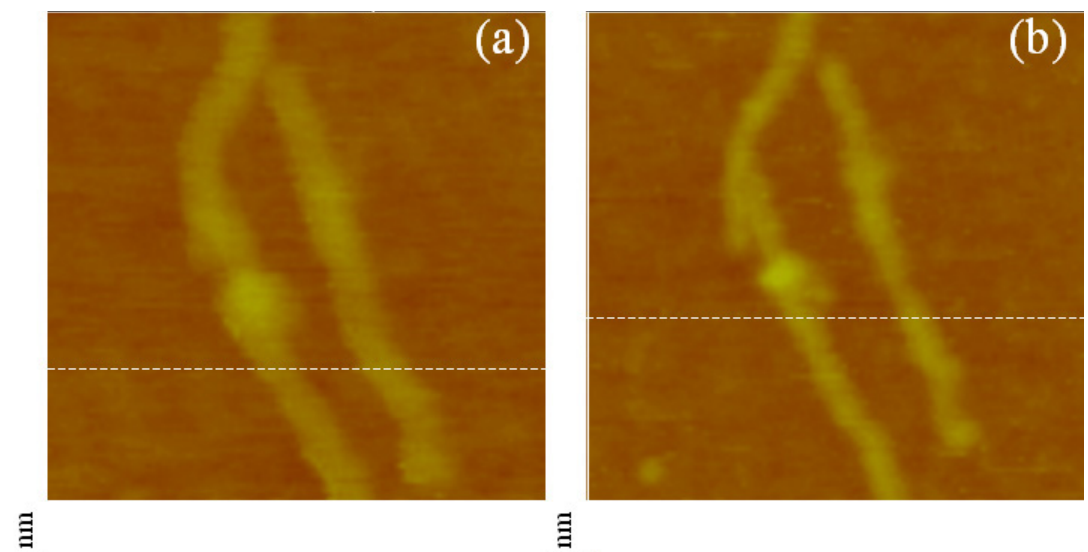

\begin{abstract}
罗
\end{abstract}
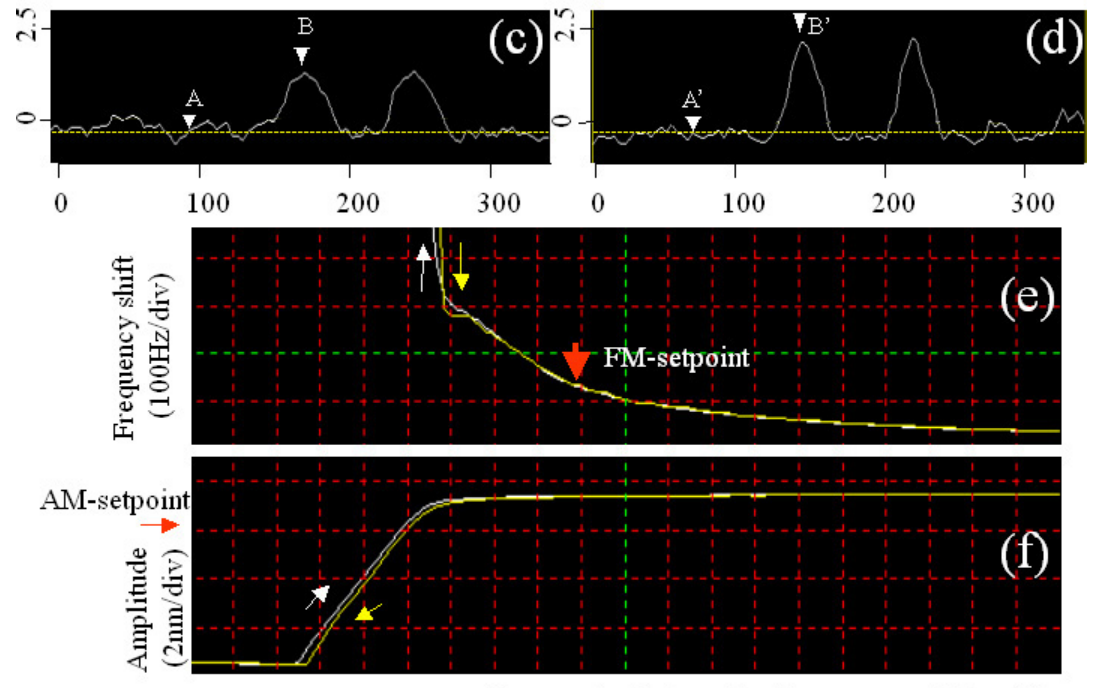

Tip-sample distance (nm)

$2.5 \mathrm{~nm} / \mathrm{div}$

Figure 3. Measurements of duplex DNA on a mica surface in water. Scan area: $350 \mathrm{~nm} \times 350 \mathrm{~nm}$. The resonance frequency of the cantilever is $\sim 36.64 \mathrm{kHz}$ and the $Q$-factor is $\sim 3.5$. (a) Topographic image taken with the $\mathrm{AM}$ mode $\left(\mathrm{A}_{\text {free }}=6.2 \mathrm{~nm}, \mathrm{~A}^{\prime}=4.8 \mathrm{~nm}\right)$. (b) Topographic image taken with the FM mode $(\Delta f=+94 \mathrm{~Hz}, \mathrm{Amp}=4.8 \mathrm{~nm})$. (c) Height profile of duplex DNA along the white line in (a). The average height of the terrace is shown with a yellow dashed line. Point A indicates a position on the line profile that intercepts the yellow line.

(d) Height profile of duplex DNA along the white line in (b). The same procedure as in (c) has been applied here to obtain the average height and point $\mathrm{A}^{\prime}$. (e) Approach/retract curve of the frequency shift versus the tip-sample distance. The setpoint of the frequency shift is indicated by a red arrow. (f) Approach/retract curve of oscillation amplitude versus the tip-sample distance measured simultaneously with (e).
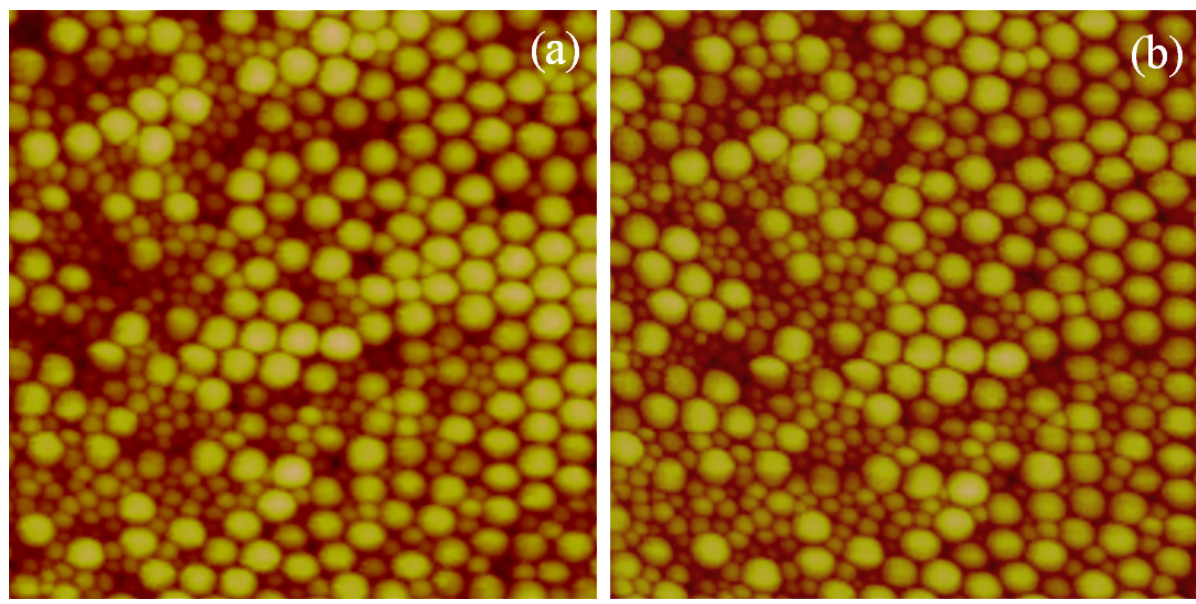

Figure 4. Topographic images of polystyrene spheres deposited on an HOPG surface taken in air. The resonance frequency of the cantilever is $\sim 86.48 \mathrm{kHz}$ and the $Q$-factor is $\sim 178$. Scan area: $750 \mathrm{~nm} \times 750 \mathrm{~nm}$. (a) AM-AFM image $\left(\mathrm{A}_{\text {free }}=9 \mathrm{~nm}, \mathrm{~A}^{\prime}=7.3 \mathrm{~nm}\right.$ ). (b) FM-AFM image $(\Delta f=-245 \mathrm{~Hz}, \mathrm{Amp}=7.3 \mathrm{~nm})$. 

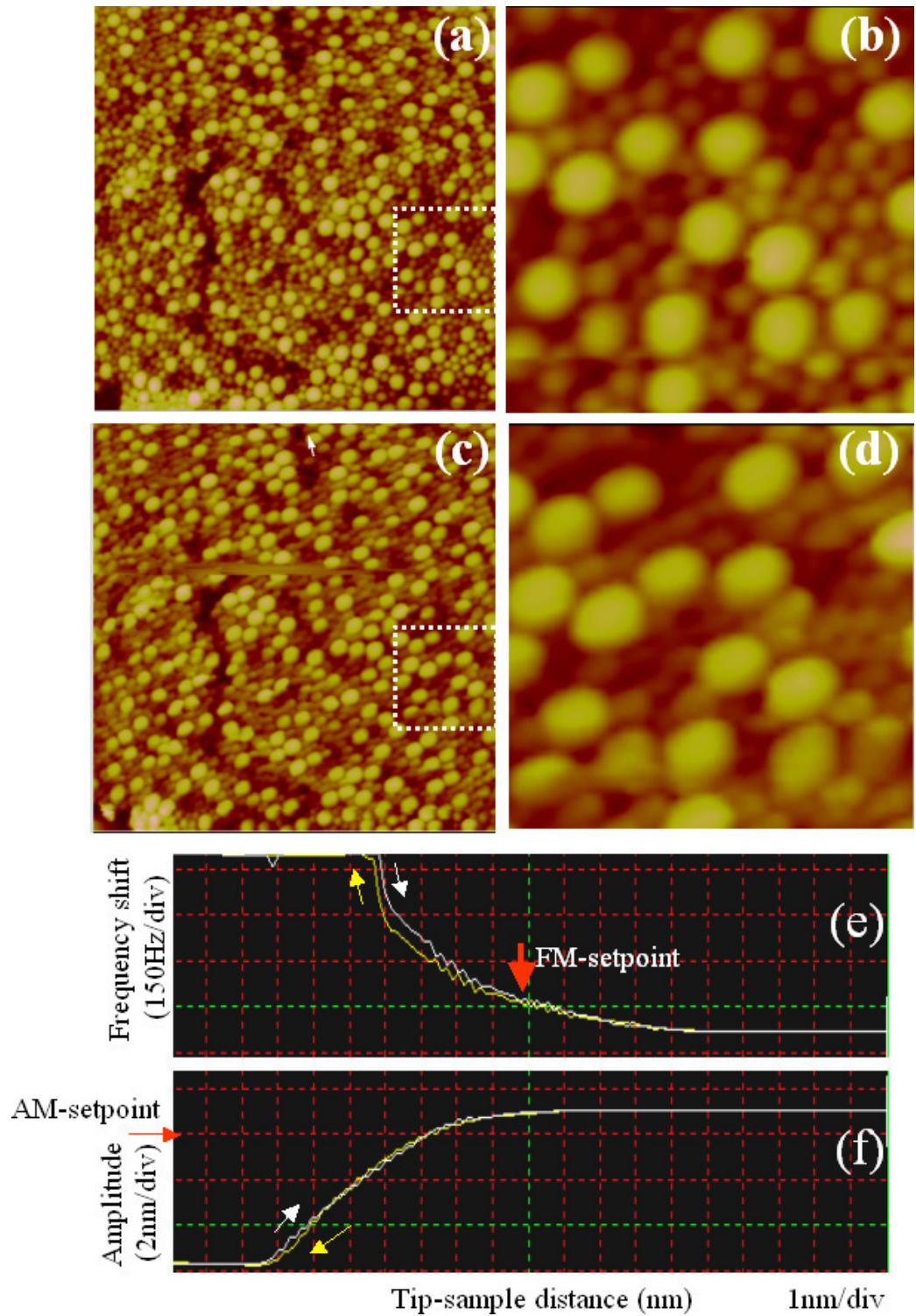

Figure 5. Measurements of polystyrene spheres deposited on an HOPG surface in pure water. The resonance frequency of the cantilever is $\sim 56.65 \mathrm{kHz}$ and the $Q$-factor is $\sim 5$. (a) Topographic image taken with the FM mode. Scan area: $1.1 \mu \mathrm{m} \times 1.1 \mu \mathrm{m} . \Delta f=+82 \mathrm{~Hz}$ and Amp $=5.8 \mathrm{~nm}$. (b) Enlarged image of the box outlined in (a). Scan area: $270 \mathrm{~nm} \times 270 \mathrm{~nm}$. (c) Topographic image taken with the AM mode $\left(\mathrm{A}_{\text {free }}=6.9 \mathrm{~nm}, \mathrm{~A}^{\prime}=5.8 \mathrm{~nm}\right.$ ). (d) Enlarged image of the box outlined in (c). Scan area: $270 \mathrm{~nm} \times 270 \mathrm{~nm}$. (e) Approach/retract curve of the frequency shift versus the tip-sample distance. The setpoint of the frequency shift is indicated by a red arrow. (f) Approach/retract curve of oscillation amplitude versus the tip-sample distance measured simultaneously with (e).

of polystyrene sphere can be discerned with these two modes. In the AM mode, we choose the free oscillation amplitude at $9.0 \mathrm{~nm}$, and the working amplitude is set at $7.3 \mathrm{~nm}$. The FM mode is also operated in the attractive regime with a constant frequency shift of $-245 \mathrm{~Hz}$. There is no significant difference between images taken with these two modes in air.

However, significant differences in the topographic images of polystyrene spheres are seen in water. Figure 5(a) shows a topographic image of the polystyrene spheres of 20 and $50 \mathrm{~nm}$ in pure water, taken with the FM mode. The enlarged image of the region outlined by white dots is shown in figure 5(b). As is the case in figure 3(b), the FM detection is operated in the repulsive regime with a constant frequency shift of $+82 \mathrm{~Hz}$. Both sizes of polystyrene sphere can be well resolved. For the images of the same area taken with the AM mode, serious distortion in the polystyrene spheres can be seen in figures 5(c) and (d). Even though the individual polystyrene spheres of $50 \mathrm{~nm}$ can be resolved, nearly all of them appear elongated. The most significant difference is seen on small polystyrene spheres of $20 \mathrm{~nm}$. They are hardly resolved with the AM mode. This demonstrates that the tip exerts a strong force on the sample in the AM mode, which also degrades the spatial resolution of the image.

Figure 5(e) shows the measured approach/retract curve of the frequency shift versus the tip-sample spacing for the FM mode. Meanwhile, the oscillation amplitude versus the distance is simultaneously recorded, as shown in figure 5(f). Similar to the case in figure 3 , there is no attractive regime 
in water. The resonance frequency exhibits a gradual increase for the initial approach and a sharp increase below a certain tip-sample separation. The approach/retract curve shows little hysteresis. Figure 5(f) indicates that the oscillation amplitude does not change in the initial approach even though the frequency shift has exhibited a gradual increase for several nanometres. Around the position of our set point in the frequency shift, a gradual decrease in the amplitude starts to occur. Obviously, the oscillation amplitude is not as sensitive to the tip-sample interactions as the frequency shift.

One may wonder why the frequency shift is much more sensitive to the tip-sample interactions than the amplitude change in water. The $Q$-factor is significantly reduced in liquid. The broad peak for the amplitude versus the driving frequency indicates that the oscillation amplitude of the cantilever changes little over a wide frequency range. With the advanced detection of the frequency shift using the phaselocked-loop circuit, the weak tip-sample interactions can cause a detectable change in the resonance frequency. However, the oscillation amplitude does not show any appreciable change with such a small shift in the resonance frequency due to the low $Q$-factor. For a detectable change in the amplitude, the tip-sample interactions should be very strong, which also causes a large resonance frequency shift. This would lead to the deformation of soft samples and the relatively poor image resolution for the AM mode.

In air, the tip operated with the FM mode in the attractive regime exerts a more gentle force on samples than the AM mode. However, for a stable operation of the FM mode in the attractive regime, one should be very careful about the scanning speed, the roughness of the sample, and vibration problems of the environment. If the tip accidentally jumps into the repulsive regime, the tip would crash into the sample due to the instability of the feedback control. For operation with the AM mode, the monotonically decreasing oscillation amplitude as a function of the reducing tip-sample distance would ensure the stable operation of the feedback at all tip-sample distance.

In water, the FM mode can be operated only in the repulsive regime. Then, why does the tip cause little deformation of DNA molecules in water? Both figures 3(e) and 5(e) exhibit a gradual increase in the resonance frequency for at least several nanometres, which is much larger than the height of DNA molecules. This suggests that the initial repulsive interactions are not related to the physical contact of the AFM tip with the sample. The repulsive interactions may results from the many complicated interactions in liquid, such as electrostatic forces and hydration forces [19]. The study of these interactions would be an important subject for further investigation. The position at which a sharp increase of the resonance frequency occurs (see figures 3(e) and 5(e)) may be closer to the contact point between the tip and the sample. Therefore, the operation of the FM mode in water as shown in this work may still be in the non-contact regime.

We note that our measurements of the amplitude versus the separation measured with the constant excitation in the FM mode, as in figures 2(f), 3(f), and 5(f), are not the same as the amplitude measurement in the AM mode. We do not make this measurement with the AM mode because it cannot be obtained simultaneously with the frequency shift.
Measurements with the two modes can only be carried out in different approach/retract cycles. However, a small and unknown displacement in the tip-sample separation would be introduced because of thermal drifts and the nonlinear and time-varying properties in the response of piezoelectric materials. Since our AFM is operated in the low-force regime (low-frequency shift and amplitude change), we expect that the amplitude change taken with the FM mode in this regime might be very similar to the amplitude change taken with the AM mode, as long as the $Q$-factor is not very high. For the case in water, the $Q$-factor is usually smaller than 10 . The oscillation amplitude of the cantilever is not very sensitive to small resonance-frequency shift.

In the literature, there were few works that have compared the performance of the AM mode (or the tapping mode) and the FM mode (or the NC-AFM mode) in imaging soft materials on surfaces. Maeda et al imaged single- and double-stranded DNA using the AM and the FM modes [20]. They reported that the NC-AFM mode revealed detailed structures that could not be seen in the tapping mode, which is similar to our finding presented here. However, their experiments for the two AFM modes were carried out in two different environments with two different types of AFM cantilever. The tapping mode was done in air, but the NC-AFM was in an ultra-highvacuum environment. Therefore, it was not clear whether the resolution difference was caused by the different detection schemes, by the different environments, or by the different AFM cantilevers.

Sekiguchi et al modified a commercial AFM by the addition of a self-oscillation circuit [21]. They imaged assembled tau protein in liquid and compared the frequency feedback and the amplitude feedback with their self-oscillation method. The frequency feedback imaging is similar to our case of the FM mode. They reported that the structure of protein fibres was clearly imaged with this mode. No damage was observed and the deformation was negligible. They estimated that the contact force was less than $100 \mathrm{pN}$. In contrast, the proteins were destroyed using the amplitude feedback imaging, indicating a much stronger vertical force on the proteins. They used the same tip and imaged the same area under the same environment for both detection schemes. They concluded that the FM feedback was more sensitive than the amplitude feedback, similar to our conclusion. However, their amplitude feedback was carried out with the self-oscillation method, which is slightly different from the tapping mode with which the driving frequency is fixed at a working frequency during scanning. Nevertheless, they had shown that the FM feedback applied very low forces on soft materials in liquid.

\section{Conclusions}

An AFM tip operated in frequency-modulation (FM) mode exerts a very gentle force on soft materials and this mode provides a height measurement close to the true value. A tip operated in the amplitude-modulation (AM) mode exerts a stronger force on soft samples and may cause their deformation, especially in a liquid environment. The resolution of the FM mode is about the same as that of the AM mode for operation in air, but the former is significantly superior to the 
latter in water. Our study clearly shows that the frequencyshift detection scheme is more sensitive to the tip-sample interactions than the amplitude detection scheme, especially in water. The much reduced $Q$-factor of the cantilever in water may be responsible for the much better sensitivity of the FM detection than the AM detection. Therefore, the FM mode can provide a sufficient sensitivity to image the true and fine structure of soft matter on surfaces.

\section{Acknowledgments}

This research is supported by the National Science Council of ROC (contract No NSC94-2120-M-001-006) and Academia Sinica.

\section{References}

[1] Morita S, Wiesendanger R and Meyer E 2002 Noncontact Atomic Force Microscopy (Berlin: Springer)

[2] Giessible F J 1995 Science 26768

[3] Hansma P K et al 1994 Appl. Phys. Lett. 641738

[4] Albrecht T R, Grütter P, Horne D and Rugar D 1991 J. Appl. Phys. 69668

[5] Giessible F J 2003 Rev. Mod. Phys. 75949
[6] Sugawara Y, Ohta M, Ueyama H and Morita S 1995 Science 2701646

[7] Lantz M A et al 2001 Science 2912580

[8] Eguchi T and Hasegawa Y 2002 Phys. Rev. Lett. 89266105

[9] Eguchi T et al 2004 Phys. Rev. Lett. 93266102

[10] Fukuma T, Kobayashi K, Matsushige K and Yamada H 2005 Appl. Phys. Lett. 86193108

[11] Fukuma T, Kobayashi K, Matsushige K and Yamada H 2005 Appl. Phys. Lett. 87034101

[12] Fukuma T, Kimura M, Kobayashi K, Matsushige K and Yamada H 2005 Rev. Sci. Instrum. 76053704

[13] Hung S K, Hwu E T, Hwang I S and Fu L C 2006 Japan. J. Appl. Phys. 45 (3B) 1917

[14] Saenger W 1984 Principles of Nucleic Acid Structure (Berlin: Springer)

[15] Moreno-Herrero F, Colchero J and Baró A M 2003 Ultramicroscopy 96167

[16] Hansma H G, Revenko I, Kim K and Laney D E 1996 Nucleic. Acids Res. 24713

[17] Tang J, Li J, Wang C and Bai C 2000 J. Vac. Sci. Technol. B 181858

[18] Chang Y C et al 2004 Japan. J. Appl. Phys. 43 (7B) 4517

[19] Bonnel D 2001 Scanning Probe Microscopy and Spectroscopy: Theory, Techniques, and Applications (New York: Wiley)

[20] Maeda Y, Matsumoto T and Kawai T 1999 Appl. Surf. Sci. 140400

[21] Sekiguchi H et al 2003 Appl. Surf. Sci. 21061 